\title{
The Influence of PZT Actuators Positioning in Active Structural Acoustic Control
}

\author{
P. Švec, V. Jandák
}

This paper deals with the effect of secondary actuator positioning in an active structural acoustics control (ASAC) experiment. The ASAC approach is based on minimizing the sound radiation from structures to the far field by controlling the structural vibrations. In this article a rectangular steel plate structure was assumed with one secondary actuator attached to it. As a secondary actuator, a specially designed piezoelectric stripe actuator was used. We studied the effect of the position of the actuator on the pattern and on the radiated sound field of the structural vibration, with and without active control. The total radiated power was also measured. The experimental data was confronted with the results obtained by a numerical solution of the mathematical model used. For the solution, the finite element method in the ANSYS software package was used.

Keywords: ASAC, Active radiation control, vibrations, sound radiation.

\section{Introduction to ASAC}

Active Structural Acoustics Control (ASAC) is a treatment for reducing the low-frequency sound radiated by a structure to the far Weld. This method is a combination of two other methods - Active Noise Control (ANC) and Active Vibration Control (AVC). The object of ASAC is similar to that of ANC but the control mechanism is closer to that of AVC. All of these methods use secondary actuators to ensure the desired control criterion - ANC uses sound sources, while AVC uses mechanical force sources. The object of AVC is to minimize (locally or globally) the vibration pattern of a controlled structure $[3,5]$. This could increase the total radiated power due to the fluid structure coupling phenomenon. Thus it is not applicable when radiated sound is at the centre of our interest. ANC utilizes the destructive interference of sound waves to achieve its object. This creates zones of quiet in the original sound field, but the overall energy density increases. In cases when we want to reduce radiated sound globally or reduce the total radiated power, other control strategies (e.g. ASAC) must be applied.

The total power $\Pi$ radiated by a planar source, e.g., a vibrating panel, is proportional to the normal velocity of its surface, and can be expressed as

$\Pi(\omega)=\frac{\rho_{0} c_{0} k}{8 \pi^{2}} \mathfrak{R}\left[\int_{-\infty-\infty}^{\infty} \int_{-\infty}^{\infty} \frac{\left|V_{z}\left(k_{x}, k_{y}\right)\right|^{2}}{\sqrt{k^{2}-k_{x}^{2}-k_{y}^{2}}} \mathrm{~d} k_{x} \mathrm{~d} k_{y}\right]$,

where $k$ is the wave number vector magnitude, $k_{x}, k_{y}$ are its components in the $x$ and $y$ directions, $\rho_{0}$ and $c_{0}$ are air density and phase speed of sound respectively, $V_{z}$ is the $2 \mathrm{D} \mathrm{k}$-space Fourier transform of the normal velocity of the vibrating surface, and $\Re(\cdot)$ means the real part of its argument. Therefore by controlling the distribution of the normal velocity over the surface we can control the radiated power. This is the basic idea of ASAC, which acts mechanically on the surface of a vibrating body to change its vibration pattern.

\section{Flexural waves in thin plates}

Let us consider a simple situation - a thin plate radiating sound due to its vibrations. If the thickness of the plate is negligible in comparison with its other dimensions, the most important type of waves (in terms of sound radiation) are flexural waves. Thus we have to solve a twodimensional elastic

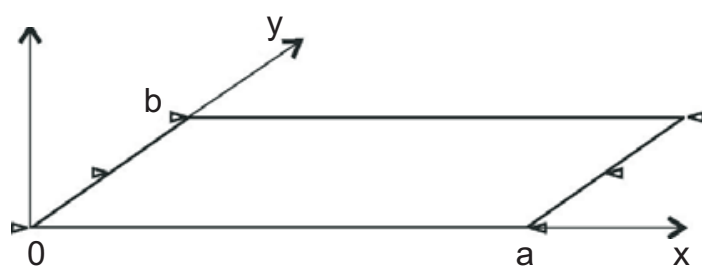

Fig. 1: Coordinate system orientation of the plate.

motion problem. According to thin-plate theory [2, 6], the equation of motion in such a case is

$$
E I \nabla^{4} u+\rho h \frac{\partial^{2} u}{\partial t^{2}}=-p(x, y, t) .
$$

Where $E$ is Young's modulus, $I$ is the moment of inertia per unit width, $h$ is thickness of the plate, $\rho$ is density of the plate, and $p(x, y, t)$ is a general load function (in units of force per unit area). The biharmonic operator $\nabla^{4}$ can be expanded in a Cartesian coordinate system into the following form

$$
\nabla^{4} u=\frac{\partial^{4} u}{\partial x^{4}}+2 \frac{\partial^{4} u}{\partial x^{2} \partial y^{2}}+\frac{\partial^{4} u}{\partial y^{4}} .
$$

Let us consider a rectangular plate clamped on the two shorter sides and free on the other two sides, see Fig. 1. We can write boundary conditions [1] for the clamped sides (at $x=0$ and $x=a$ )

$$
\frac{\partial^{2} u}{\partial x^{2}}=0, \quad \frac{\partial^{3} u}{\partial x^{3}}=0
$$

and for the free sides (at $y=0$ and $y=b$ ) 

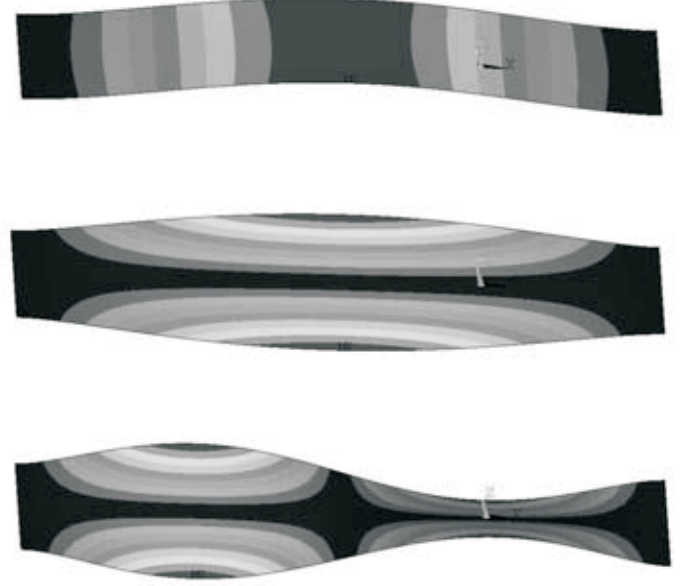

Fig. 2: Shape of the first six modes of the plate

$$
u=0, \quad \frac{\partial u}{\partial y}=0 .
$$

At the beginning of our analysis, the free vibrations of the plate were solved to obtain modes of the system. For the solution, the ANSYS software package was used. Fig. 2 shows the shapes of the first six modes.

Table 1: Frequencies of the first six modes of the plate

\begin{tabular}{|c|c|c|c|c|c|c|}
\hline mode & 1 & 2 & 3 & 4 & 5 & 6 \\
\hline$f[\mathrm{~Hz}]$ & 27.6 & 77.2 & 99.7 & 153 & 206 & 255.7 \\
\hline
\end{tabular}

\subsection{Forced vibration - shaker load function}

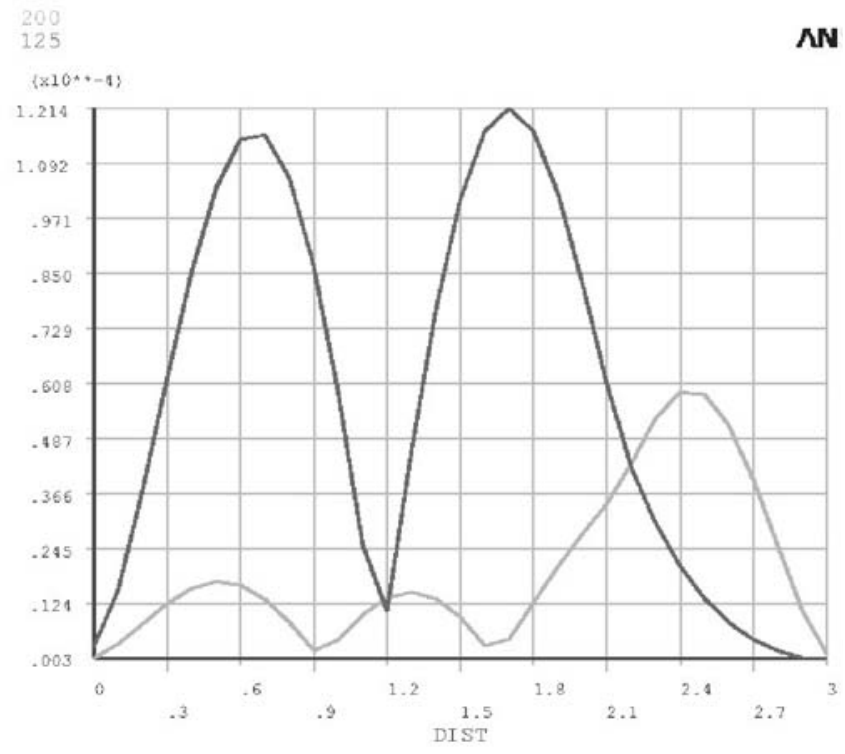

Fig. 3: Forced vibrations of the plate (point-force actuation at $x=215 \mathrm{~mm}), f=125$ and $200 \mathrm{~Hz}$, solved by ANSYS

Let us assume forced vibrations of the plate caused by a harmonic point force source. This condition appears on the right side of equation (2), and it should be written using the Dirac function as

$$
f(x, y, t)=F \delta\left(x-x_{i}\right) \delta\left(y-y_{i}\right) \mathrm{e}^{j \omega t},
$$
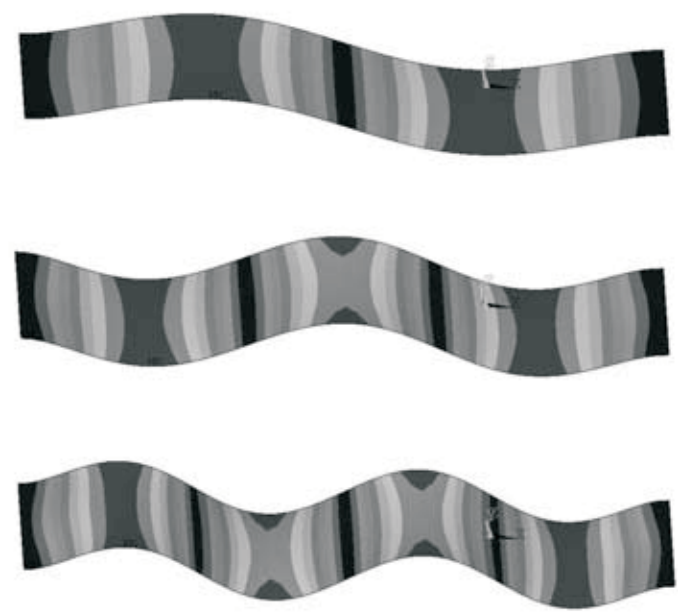

where $x_{i}$ and $y_{i}$ are coordinates where the force is applied and the force amplitude $F$ has units of force per unit area.

\subsection{Secondary actuator - load function}

To control sound radiation, secondary actuators have to be applied. For the experiment, an actuator was constructed based on piezoelectric stripe actuators. The stripe actuator was produced by APC International, Ltd [7]. It is a piezoceramic (PZT) bending actuator. The actuator consists of two thin strips of piezoelectric ceramic bonded together, with the direction of polarization coinciding. Electrically, the strips are connected in parallel. When an electrical input is applied, one ceramic layer expands and the other contracts, causing the actuator to flex. The stripe actuator has a relatively small blocking force (approximately 0.1-1 N) but a large displacement. The maximum displacement is up to $2 \mathrm{~mm}$ when the actuator is fixed in a cantilever mounting configuration. The first resonant frequency of the selected PZT strip (Catalog No. 40-1040 [7]) was $70 \mathrm{~Hz}$. To optimize the effect of the stripe actuators, they were clamped to the metal frame in a cantilever mounting. The frame was then attached directly to the steel plate, see Fig. 4. As the piezos oscillate, the bending moment

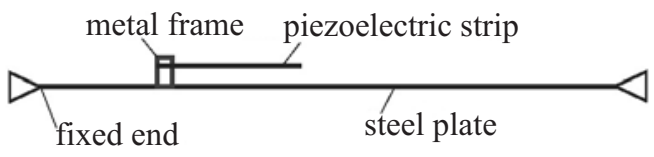

Fig. 4: Schematic cross-section of the actuator design and mounting on the plate

is revealed at the clamped end of the PZT actuator. This moment is transmitted through the frame to the primary structure, acting as a line moment load function [2]. The effect of the added mass of the frame and PZT actuators is almost negligible when active control is turned on, as will be shown below.

To describe the effect of the line bending moment acting in the $y$-axis direction, we should add another term to the right side of equation (2)

$$
m(x, y, t)=M \delta^{\prime}\left(x-x_{k}\right) \mathrm{e}^{j \omega t} .
$$

The symbol $\delta^{\prime}(x)$ means derivative of the Dirac delta function with respect to its argument, and $M$ is the moment ampli- 


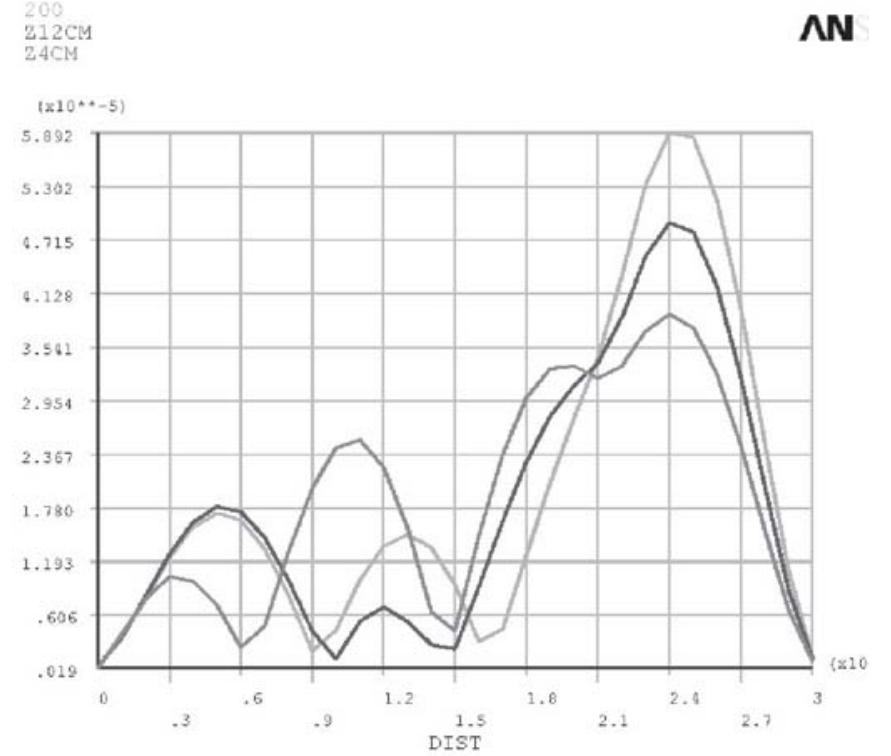

Fig. 5: Theoretical plate displacement values along the $x$-axis by the FEM model for single point force actuation and for two positions of the secondary actuator $(x=4$ and $12 \mathrm{~cm}$ ), active control is off

tude (in units of moment per unit length). Finally, we have to solve the equation

$E I \nabla^{4} u+\rho h \frac{\partial^{2} u}{\partial t^{2}}$
$=F \delta\left(x-x_{i}\right) \delta\left(y-y_{i}\right) \mathrm{e}^{j \omega t}-M \delta^{\prime}\left(x-x_{k}\right) \mathrm{e}^{j \omega t}$.

\section{Experimental setup}

All experiments were performed in an anechoic room in the acoustical laboratory of the Department of Physics, Czech Technical University in Prague, Faculty of Electrical Engineering. In the experiments, a baffled rectangular steel plate was used. The dimensions of the plate were $300 \times 60 \times 0.5 \mathrm{~mm}$ and the boundary conditions were as described in the previous sections. An electrodynamic shaker was used as a point force actuator. It was mounted on the plate $215 \mathrm{~mm}$ from the

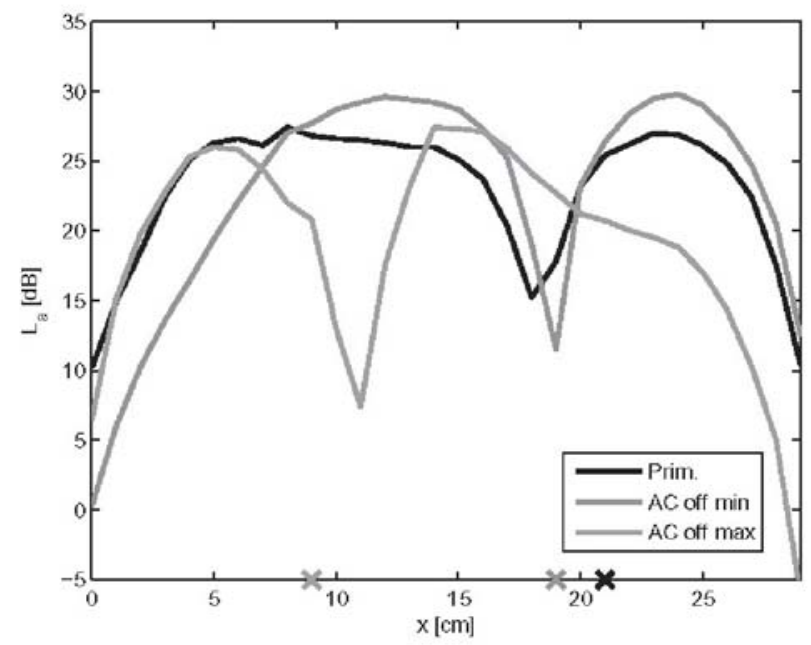

Fig. 6: Effect of the added mass of the secondary actuator on the system response - without the actuator, and with it placed on the node and antinode at $f=125 \mathrm{~Hz}$

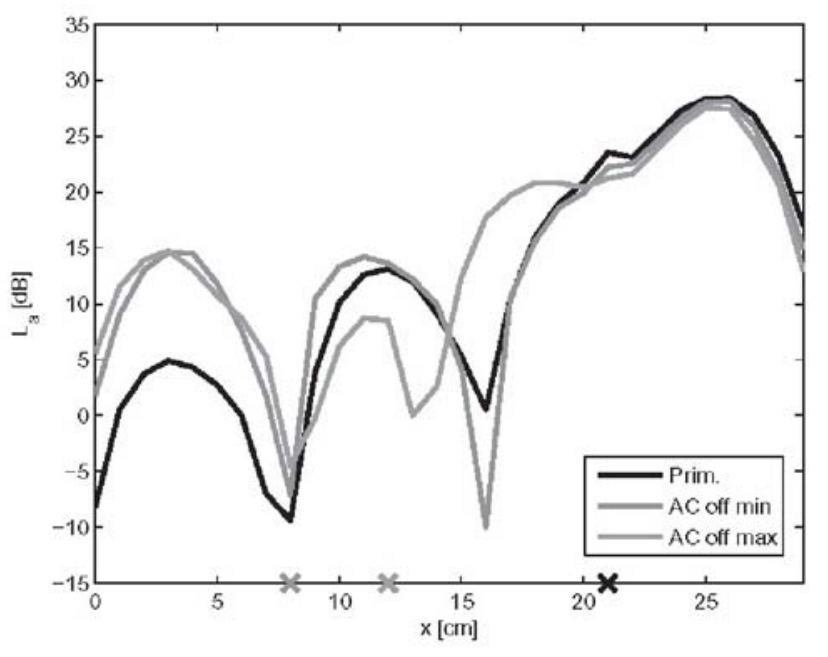

Fig. 7: Effect of the added mass of the secondary actuator on the system response - without the actuator, and with it placed on the node and antinode for $f=200 \mathrm{~Hz}$

origin in the $x$-direction and $30 \mathrm{~mm}$ in the $y$-direction (the orientation of the plate in the coordinate system is shown in Fig. 1). Only pure harmonic excitation was used. The frequencies selected for our experiments were 125 and $200 \mathrm{~Hz}$. The structural vibrations of the plate were measured along the $x$-axis, in the center-line of the plate for both frequencies and various positions of the secondary actuator (node, antinode). In this first experiment, the active control was set to off, so only the effect of the added mass on the specific locations was observed. Of greatest importance, of course, was the case when the atuator was placed directly on the antinode. In such cases, the vibration pattern was changed. Typically a new local node arises close to the actuator position, see Fig. 6 and Fig. 7.

This result corresponds well with the theoretical observations provided by the FEM model, see Fig. 5. The effect of the added mass on the sound radiation was within $\pm 3 \mathrm{~dB}$. Compared with the effect of active control, this was negligible. Active control was able to reduce the radiated sound pressure level by more than $30 \mathrm{~dB}$ with harmonic excitation.

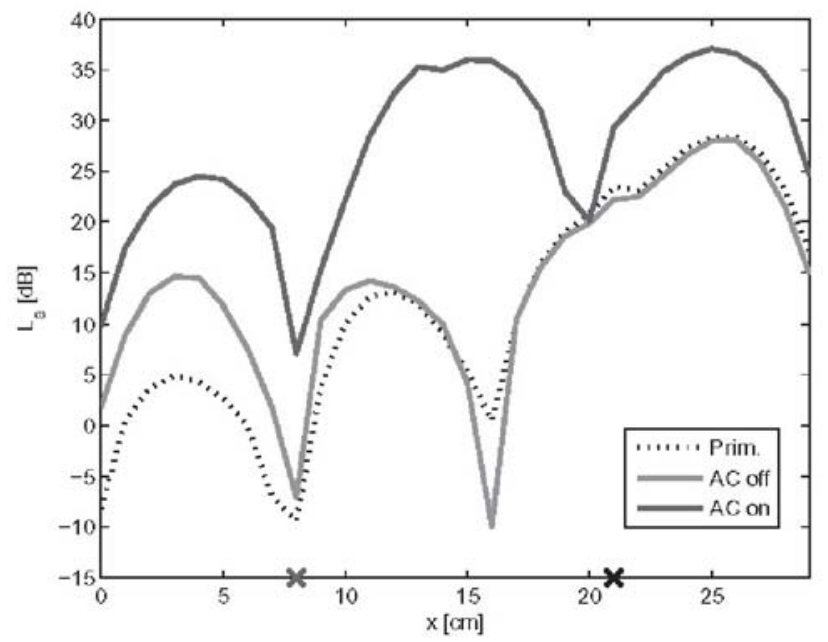

Fig. 8: Vibration pattern for $f=200 \mathrm{~Hz}$ with active control (AC on), actuator placed on the node - marked by the hatched cross on the $x$-axis, the black cross marks the position of the shaker 


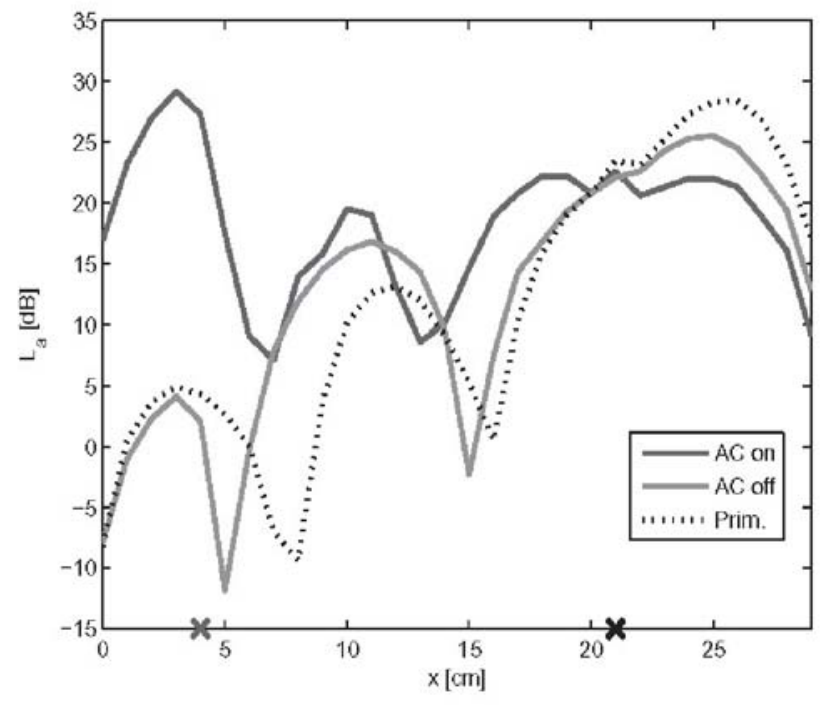

Fig. 9: Vibration pattern for $f=200 \mathrm{~Hz}$ with active control (AC on), actuator placed on the antinode - marked by the hatched cross on the $\mathrm{x}$-axis, the black cross marks the position of the shaker

In Figs. 8 and 9, the actuator was placed on the node and antinode, respectively. The vibration pattern was measured with the active control turned off (AC off) and then with the active control turned on (AC on). The dashed curve shows the forced vibrations of the primary structure without the actuator mounted on it. The displacement amplitudes increased overall, while the minimum sound pressure level was observed. This result is in good accordance with fluidstructure coupling theory and with the theoretical discussion presented in this paper.

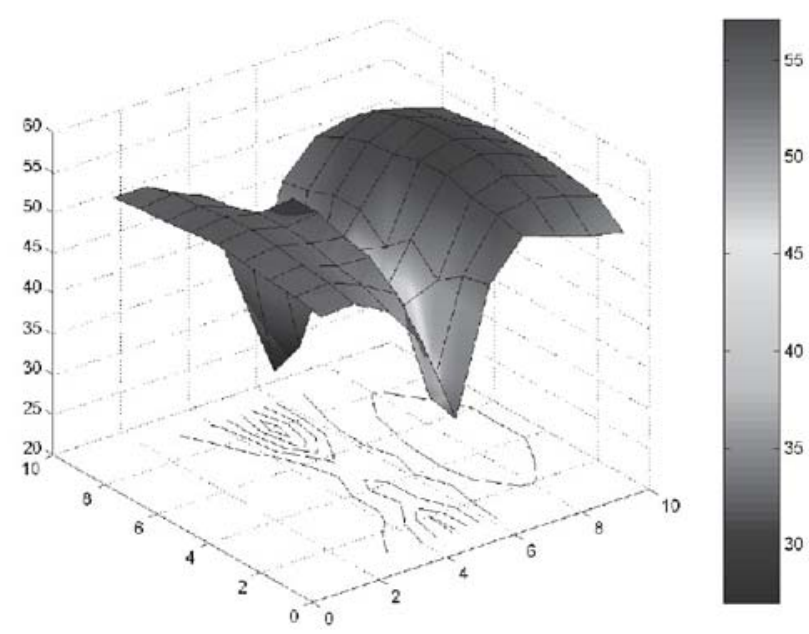

Fig. 10: Sound field $30 \mathrm{~cm}$ above the baffled plate at frequency $200 \mathrm{~Hz}$, active control is turned on

\section{Conclusion}

This paper presents theoretical and experimental studies of thin plate vibrations and the effect of an added secondary actuator which controls the structural response of the system to minimize radiated sound. The results show that a piezoelectric stripe actuator attached to the plate in a cantilever mounting is able to control the radiation effectively and almost independently of its position. The mass added to the plate (due to the actuator mounted on it) has its most significant effect on the mechanical response when placed between two nodal lines (on the maximum of the standing wave antinode). In this case, the vibration pattern is changed and a new nodal line arises close to the position of the mounted actuator. This conclusion corresponds well with the FEM model results. However, when the position of the actuator mounting is on the nodal line, the vibration pattern stays the same, as we had expected. When active control is turned on, both positions of the actuator lead to a decrease in the sound pressure level. The total radiated power decrease, measured with an intensity probe, is $10 \mathrm{~dB}$.

\section{Acknowledgments}

The research reported in this paper was supervised by Prof. O. Jiříček, FEE CTU in Prague, and was partially supported by research program MSM6840770015.

\section{References}

[1] Brdička, M., Samek, L., Sopko, B.: Mechanika kontinua, Academia, Praha, 2000, p. 358-371.

[2] Fuller, C. R., Elliot, S. J., Nelson, P. A.: Active Control of Vibration, Academic press, London, 1997.

[3] Johnson, B. D., Fuller C. R.: Broadband Control of Plate Radiation Using Piezoelectric, Double-Amplifier Active-Skin and Structural Acoustic Sensing. Journal of the Acoustical Society of America, Vol. 107 (1999), No. 2, p. 876-884.

[4] Wiliams, E. G.: Fourier Acoustics Sound Radiation and Nearfield Acoustical Holography, Academic press, London, 1999.

[5] Fuller, C. R.: Active Control of Sound Radiation from Structures: Progress and Future Directions. Active 2002, Southampton.

[6] White, R. G., Walker, J. G.: Noise and Vibration, Ellis Horwood Publishers, Chichester, 1982.

[7] APC International Ltd.: Piezoelectric Ceramics: Principles and Applications, Mackeyville, USA, 2002.

Ing. Petr ̌̌vec

e-mail: svecp1@fel.cvut.cz

Ing. Vojtěch Jandák

e-mail: jandav1@fel.cvut.cz

Department of Physics

Czech Technical University in Prague

Faculty of Electrical Engineering

Technická 2

16627 Praha 6, Czech Republic 